\title{
VARICES Y ANGIECTIDS DURANTE EL EMBARAZO *)
}

\author{
Doctor Alvaro Rugeles V. (\%)
}

Várices y angiectids son diferentes entidades que se deben diferenciar durante el embarazo. Las angiectids serán consideradas al final de este trabajo.

\section{I.-Nota históríca}

El reconocimiento y el tratamiento de las venas varicosas es tan antiguo como es la literatura médica, remontándose al siglo IV antes de Jesucristo. Aún algunos de los procedimientos quirúrgicos como también de los conservaclores usados hoy día, han tenido sus orígenes en técnicas que fueron descritas hace varios siglos.

Un gran número de ingeniosos procedimientos conservadores y quirúrgicos han sido empleados para el tratamiento de los pacientes varicosos, a saber: incisiones, punzadas múltiples, división, presión, cauterio, ligaduras múltiples con o sin sección, resección, excisión, ablación, extirpación, extracción, flebo-extracción, flebectomía, arrancamiento, avulsión, ligaduras múltiples percutáneas (a través de la piel), irradiación, astringentes y escarificantes aplicados a la piel que cubre la várice con el objeto de producir trombosis. Compresión de las várices por medio de venda_ jes y de medias empleando lino, piel de perros, cuero, algodón, iana, caucho, yeso, substancias plásticas, medias elásticas. Una gran variedad de soluciones trombo-esclerosantes intravenosas han sido empleadas y diversas ingeniosas técnicas para su inyección,

(*) Trabajo leído en la reunión de la Sociedad Colombiana de Obstetricia y Ginecología del mes de diciembre de 1958 (para, optar al título de miembro afiliado).

(**) Universidad Javeriana. Bogotá, Colombia.-The Graduate School of Medicine of the University of Pennsylvania, Philadelphia.-Curso en Obstetricia y Ginecología. Septiembre 1957 a mayo 1958). 
como es la inyección retrógrada. Hilos de seda, de algodón o sintéticos, dejados permanentemente dentro de la luz de la vena safena varicosa, han sido empleados para ocasionar trombosis. Compinación de ligadura quirúrgica e inyección esclerosante retrógrada por medio de un catéter introducido dentro de la luz de la vena.

Obstrucción de las venas por cicatrización y trombosis han sido los propósitos de estos métodos (5, etc.). Resultados pobres se han obtenido y el método ha sido abandonado por otro que tiene influencia por un tiempo con resultados similares al final (17). Aún ni el esclerosante ideal ni la operación ideal han sido descubiertos, y la literatura moderna de las venas varicosas muestra cegreso a los métodos históricos; "El futuro es solamente el pasa(1o nuevamente" (Foote, 5).

Arrancamiento combinado con ligaduras múltiples es actualmente la operación establecida, mejor que ninguna otra en el pasado, pero la perfección no se ha alcanzado (17). Si bien los tratamientos modernos le ofrecen al paciente alivio o curación, en la mayoría de los casos; los problemas de las várices aún persisten y están lejos de ser resueltos (5).

\section{II.-Consideraciones anatómicas}

Como los signos, síntomas y complicaciones de las venas varicosas son el resultado directo de la anatomía alterada, una breve revisión de la anatomía normal y de la fisiología será presentada. La eficacia en el tratamiento exige el uso de medidas en relación con la anatomía y la fisiología (13). Las venas del miembro inferior deben subdividirse en tres grupos funcionales:

A-Venas profundas, situadas entre la fascia profunda y el hueso, siguen el curso de las arterias principales y son las siguientes: tibial anterior y tibial posterior, las cuales desembocan en la poplítea-femoral.

B.-Venas comunicantes, también llamadas venas perforantes, están inconstantemente localizadas y son extremadamente importantes en el papel de conectar las venas profundas con las venas superficiales durante su ascenso en el miembro inferior.

C.-Venas superficiales, situadas en el tejido subcutáneo en tre la piel y la fascia profunda e independientes do las arteria. Las principales venas superficiales son: 
1. La vena safena larga (grande, interna o vena safena magna), la vena más larga en el cuerpo, extendiéndose desde el arco venoso dorsal del pie y situada anterior e internamente, cruza anteriormente el maléolo interno, la pantorrilla y el muslo, y desemboca a la vena femoral en la ingle, debajo del ligamento inguinal.

2. La vena safena corta (pequeña, menor o vena safena parva), es la continuación de la vena marginal por detrás del maléolo externo y asciende por detrás de la pierna, hasta el pliegue de ia rodilla, en donde desemboca en la vena poplítea después de perforar la fascia.

3. Veria safena accesoria.-Presente en 2 o 3 por 100 de la población (16). Drena la parte superficial media y posterior del muslo y desemboca en la vena safena larga (5).

Todas las venas del miembro inferior tienen válvulas, las cuaies son más numerosas que en las venas del miembro superior y mas numerosas en las venas profundas que en las superficiales. El promedio de distribución de las válvulas es el siguiente: tibial anterior, alrededor de 11; tibial posterior, 8 a 20 ; poplítea-femoral, 2 a 9; safena corta, 9 a 10; safena larga, 12 a 18; femoral-ilíaca raramente y no competente; vena cava inferior, ninguna. Casi todas las válvulas son bicúspides, con una valva anterior y otra posterior. Raramente una válvula sencilla o una tricúspide (9).

Las primeras válvulas en la vena safena tienen su origen en el feto humano aproximadamente entre los tres y los cinco meses. Durante los dos meses siguientes otras se desarrollan, pero es probable que al final del quinto mes de vida intrauterina el total de las válvulas ha sido establecido (1).

Es significante, aunque puede ser solamente una coincidencia, que las válvulas son colocadas primariamente en áreas de actividad muscular y en la época en que el feto comienza a ejecutar movimientos del esqueleto; mientras que el órgano intrínseco del mecanismo propulsivo, el corazón, es establecido más temprano (1).

La coincidencia de las válvulas no está cercanamente relacionada con la postura del animal. La existencia de las válvulas está en directa relación con la cantidad de músculo de acuerdo con los estudios comparativos en monos macacus rhesus, perros y gatos (12). La dirección normal de la corriente sanguínea es de las venas superficiales a través de las ramas comunicantes, las cuales perforan la fascia y desembocan en las venas profundas, siendo la dirección de la corriente hacia el corazón. 


\section{III.-Factores etiológicos}

Basados en los factores que son comunes en las venas variosas, nosotros podemos agrupar los factores etiológicos entre primarios, secundarios y varios. Esos factores pueden estar particularmente presentes durante el embarazo..

1. Factores primarios.-Persona susceptible a las várices: a) Debida a un defecto biológico, constitucional o congénito-anatómico en la estructura del mesodermo de las venas, como puede ser una inherente debilidad de las paredes de los vasos, la llamada astenia del mesodermo; b) $\mathrm{O}$ debido a una insuficiencia congénita de las válvulas, porque ciertos factores predisponentes conducen a la formación de las várices en algunos individuos y no en otros (4), (8). El gran porcentaje de pacientes con una historia familiar de venas varicosas sugiere ese factor. Cua¿enta por ciento de todos los individuos con venas varicosas tienen historia familiar de várices (4).

Las venas varicosas pueden estar asociadas con hemorroides, várices de la vulva, adiposidad de las nalgas, muslos y piernas sugiriendo mal funcionamiento glandular, más frecuente en mujeres ,pero frecuente en hombres del tipo Froehlich. Generalmente en hombres delgados, musculados, con tejido conectivo pobre, las venas son prominentes con varicocele o hernia inguinal asociados (20).

Kampmeier dice: "Las válvulas impiden la devolución de la sangre hacia las venas inferiores; ellas, estando colocadas a intervalos, dividen la columna de sangre en pequeños segmentos, y cada válvula tiene que soportar solamente una fracción de la coiumna total. La incompetencia de las válvulas precede, no sigue, a la dilatación venosa" (1), (13).

Williams dice: "La principal función de las válvulas no es contrarrestar los efectos de la gravedad. La función de las válvulas es contrarrestar los efectos por subidas excesivas súbitas en la presión de la sangre durante la contracción muscular y proteger los capilares y las vénulas" (12).

Wrigh dice: "Las venas primero se dilatan en circunferencia. jroduciendo relativa insuficiencia de las válvulas. Después ellas aumentan en longitud y más tarde se hacen tortuosas. Hay marcada pérdida en la elasticidad, las paredes se hacen irregulares y la estructura de las válvulas se destruye" (4). 
Veal dice: "Desde que las venas varicosas son complicación común en el embarazo en todas las razas y en todas las clases, no sarece lógico considerar la existencia de una "predisposición pe"uliar", tal como una debilidad congénita de las válvulas del sistema venoso" (19).

\section{2.-Factores secundarios:}

A) Puro efecto de gravedad de la sangre hacia el pie o factor de presión hidrostática o flujo de reversa, propio del hombre a causa de su postura erecta (2). Cualquier aumento en la presión ejercida en cualquier porción de la columna de sangre del sistema de la safena (en la posición de pies), que por las leyes de Pascal es transmitida igualmente a través del sistema. La vena femorai profunda no está generalmente comprometida a causa del mecanismo protector del músculo y la dascia. El sistema de la safena, superficial y con menos adécuado soporte mesodérmico, se encuentra particularmente vulnerable a la suma del efecto de la gravedad de la sangre, más el agregado efecto de tensión (2). Así, cualquier tenisión o presión intra-abdominal inferior o pélvica, contra la vena cava inferior o venas ilíacas, es capaz de forzar las paredes de los vasos hacia afuera, como en los esfuerzos del parto, el pujo, la tos (bronquitis crónica) u otros esfuerzos. En el embavazo hay, además, aumento del volumen sanguíneo.

B) Cualquier condición anatómica, adquirida o funcional, que pueda causar suficiente obstrucción externa o interna (intraluminal) para impedir el retorno o ascenso del flujo sanguíneo en las venas de las extremidades inferiores (2), (5). Embarazo, grances tumores, obesidad abdominal, ascitis, congestión repetida del área pélvica durante la menstruación, contribuyen a la causa y a la agravación de las venas varicosas (7), (13). Presión exterior baja por vestidos estrechos, fajas estrechas, ligas, etc.

Posición y ocupación: a) prolongadas posiciones de pies: dentistas, peluqueros, ascensoristas, polic'as de tráfico, dependientes de mostrador; b) sentadas prolongadas, particularmente con los pies colgando: largos viajes en tren, automóvil o avión, cines proiongados, sastres, costureras; subir escaleras es otro factor de agravación. Las venas varicosas parece que no se desarrollan en individuos que caminan considerablemente, como en los que trabajan parados (13). Anastomosis o fístulas arterio-venosas congénitas o adquiridas que causan aumento en la presión y dilatan las venas (efecto de martilleo de los líquidos, efecto de la pul- 
sación. El efecto de la placenta es el mismo de una fístula arteriovenosa modificada, y es un factor adicional (7). La flebo-trombos1s, a causa de la obstrucción circulatoria, es otro factor.

La incidencia es más alta en las mujeres que en los hombres, excluyendo el embarazo. El embarazo agrava las venas varicosas que existan previamente. Once por cinto de las pacientes obstétricas tuvieron significantes venas varicosas en 600 pacientes estudiadas consecutivamente (8). La incidencia de las várices en el embarazo ha sido reportada entre el 11 y el 50 por 100; pero solamente un pequeño número de ellas requieren tratamiento conservador (16). Cualquier mejoría en las venas varicosas después del embarazo es solamente temporal, puesto qu @l daño permanente se ha ocasionado, y con cada sucesivo embarazo o simplemente con el envejecimiento, las várices se van haciendo más y más pronunciadas.

\section{3.-Factores varios:}

Deficiencia en vitamina $\mathrm{C}$ en la dieta (escorbuto). El efecto 12 Rutin (una substancia del factor vitamínico P) en las várices está siendo estudiado. Factores endocrinos y deficiencia en vitaminas, son factores dudosos en la etiología de las várices. Las cantidades de estrógeno y de pregnandiol fueron normales en cuatro pacientes con venas safenas varicosas (15). La progesterona no tiene efecto sobre el tono de las paredes vasculares (15).

\section{IV.-Signos y síntomas}

A) Signos-Por inspección y palpación se aprecian las várices que pueden ser de diversos tipos, longitud, tamaño y variedades, Problemais cosméticos (ver complicaciones).

B) Síntomas.-Las quejas de los pacientes con venas varicosas comprenden desde ninguna hasta una gran variedad. Algunos pacientes con enormes dilataciones venosas no tienen problemas. Otros pacientes, relativamente con pequeñas venas varicosas, se quejan notablemente. Fatiga, incomodidad o dolor en el miembro inferior después de prolongado ejercicio o de largos períodos de pie.

Sensación de llenura y de congestión en la pierna cuando se está de pies, que se agrava hacia el final de un día de actividad o durante el período menstrual. Fatiga dolorosa, calambres musculares nocturnos. Los sintomas son aliviados en posición acostada, por masaje hacia arriba, por elevación de las piernas, usan- 
do medias elásticas correctamente, caminando, ya que estas medidas ayudan a circular la sangre por gravedad, por expresión o por la presión con la contracción muscular.

\section{V.-Métodos de estudìo del paciente}

A) Historia general.-Edad, sexo, historia familiar. Averiguar si usa veitidos estrechos que produzcan constricción. Ocupación pasada y presente. Averiguar por cirugía previa de várices y si hubo sensibilidad por soluciones esclerosantes. Averiguar por constipación, bronquitis, tumores pélvicos, embarazos.

B) Examen desde el punto de vista del sistema vascular.-Una gran proporción de enfermedades vasculares son enfermedades generalizadas, lo cual se debe tener en cuenta en el estudio de cada paciente. La mayor parte de los diagnósticos puden hacerse por una historia detallada y un cuidadoso examen, sin necesidad de complicados equipos de laboratorio ni de hospitalización. Los estudios detallados sirven principalmente para aclarar o para comprobar el diagnóstico o para determinar cuantitativamente el relativo grado del daño circulatorio y de la circulación colateral. El diagnóstico correcto tiene por consiguiente tanta importancia como el pronóstico y el tratamiento adecuados.

El modo de comienzo de las várices es importante, y puede estar relacionado con embarazo, enfermedades infecciosas, accidentes, cirugía, tromboflevitis o trombosis previas. La historia de úlceras crónicas alrededor o encima del tobillo, con pigmentación y ampollas de las piernas, sugiere insuficiencia venosa (úlcera varicosa). Hemorroides generalmente están asociadas con várices y pueden significar obstrucción venosa en el sistema de la vena porta. Las paredes de los labios de la vulva y de la vagina pueden presentar marcadas várices después de una flebitis con oclusión de la vena ilíaca y de algunas de las venas pélvicas. El reflujo a través de los tributarios de la hipogástrica es característico en las regiones glútea y vulvar y no debe confundirse con distribuciones de la vena safena; sin embargo, la dilatación de las tributarias de la vena obturadora superficial pueden confundirse con las de la vena safena en la parte alta del muslo (7). Las venas varicosas se deben estudiar en relación a duración, recurrencia, previo tratamiento, localización, etc.

Quizás las complicaciones más frecuentes son las de dolor e incomodidad en las piernas. Esos, naturalmente, son términos descriptivos vagos que deben ser analizados para descartar cual- 
quier otra enfermedad vascular. Es importante saber si el dolor E'S permanente o si es solamente nocturno y si aumenta con el ejercicio y se alivia con el descanso. Se debe averiguar si el dolor se irradia hacia atrás y hacia abajo en la pierna, como es el dolor de la ciática, o si sigue el curso de la vena femoral, como es común en la trombosis venosa. Es importante saber si el dolor está limitado a las articulaciones o si está localizado en las pantorrillas, como es frecuente en daños vasculares, o si el dolor es continuo, el cual es característico de la trombosis venosa o de la claudicación intermitente producida por oclusión arterial, como on la arterioesclerosis obstructiva.

C._Examen físico: tests o pruebas. - El paciente debe estar desvestido y debe ser examinado en un cuarto con temperatura caliente y con buena iluminación. A un paciente muy nervioso se le debe dar un sedante antes de la inspección y palpación. La capacidad y competencia de la circulación venosa del miembro inferior puede determinarse por exámenes especiales y por varios tests o pruebas del estado o condiciones de la circulación.

La venografía es raramente útil. Es un procedimiento opcional que puede tener algún valor en ciertos casos, pero que puede confundir en otros.

La arteriograf $\{a$ puede ayudar en el diagnóstico de anastomosis arteriovenosas.

La determinación del contenido o saturación de oxígeno de la sangre periférica puede tener valor en algunos casos de anastomosis arteriovenosa (fístulas).

Unos pocos de los más útiles de los numerosos tests serán descritos: Test de Brody-Trendelemburg. Test de Perthes. Test de Ochsner-Mahorner-Perthes. Estos tests fueron descritos y modificados por esos médicos y son bien conocidos. Ellos pueden tener algún valor en la determinación del estado valvular de las venas del miembro inferior, pero no son prácticos, no son satisfactorios y es frecuente la confusión en la interpretación de los resultados de esas pruebas (2), (6). Los siguientes tests son de simple ejecución:

1. Una ayuda diagnóstica para determinar el estado de las venas profundas y que por consiguiente sirve de guía para determinar cuáles casos pueden tener influencia favorable con medias elásticas. Comprimiendo las venas superficiales, se producirá iensión e incomodidad en la pierna después de 15 minutos si las venas nrofundas están obstruidas $y$, por consiguiente, la cir- 
culación profunda inadecuada. En presencia de venas profundas patentes, generalmente la pierna permanece confortable por tres horas o más. Medias elásticas pueden usarse para esta prueba de compresión (13).

2. Test de Adams.-Con el paciente en posición de pies y con los dedos de la mano con que palpa el médico, puestos sobre la desembocadura de la vena safena, se puede sentir la corriente de reflujo, como un "tremor", cuando el paciente tose o hace esfuer20 (2).

3. El método de Time-Honored o de compresión con un vendaje semielástico y con el paciente caminando vigorosamente por un determinado período de tiempo, para determinar el estado de la circulación venosa profunda. Si los sintomas son aliviados, un buen resultado puede pronosticarse para practicar la flebectomía.

4. Test de Schuartz-Homans.-Si las válvulas de la vena safena son incompetentes, con los dedos puestos sobre la porción proximal de ella, se siente el impulso sanguíneo cuando el tronco de la vena safena o las várices por debajo de ella son golpeadas con un dedo de la otra mano (7).

\section{VI.-Tratamiento del paciente varicoso. Medidas preventivas}

Moderada actividad, descanso, elevación de los miembros interiores y soporte exterior. Períodos de descanso y de elevación de las piernas en los intervalos de las horas de trabajo, proporcionan alivio. Ejercicios de las piernas son necesarios durante el puerperio. La parte de los pies de la cama de los pacientes varicosos debe ser elevada 25 a 30 centímetros, para disminuir por gravedad la presión venosa de los miembros inferiores, durante las horas de sueño.

El uso de un vendaje elástico hasta nivel de la rodilla o de medias elásticas permanentemente cuando el paciente ha de estar de pies por un largo período y evitar paradas prolongadas, previenen la agravación de las várices. Caminar durante las paradas de los vehículos en los viajes prolongados. Evitar la constricción por vestidos estrechos. Eliminar los tumores y otras causas obstructivas en la pelvis y en el abdomen. El traumatismo de las venas varicosas es una de las causas de producción de flebitis. La protección de las venas varicosas se puede obtener con el uso rutinario de medias o de vendajes elásticos. Algunos pacientes tienen complicaciones de escorbuto clínico o subclínico. Está indi- 
cada la inclusión diaria de unos 300 miligramos de vitamina C cristalina, oral, en la comida (papas frescas, cebollas, jugo de limas). Es conveniente hacer pruebas de fragilidad capilar y estudios de cantidades de vitamina $\mathrm{C}$ en la sangre. La cantidad normal de ácido ascórbico en el plasma es de 0.7 a 1.5 miligramos \%.

\section{VII.-Complicaciones en el paciente varicoso}

Flebitis. La trombosis venosa en pacientes varicosos tiene una incidencia de $33.3 \%$ (8). Embolismo pulmonar. Edema complicado con infección o con dermatitis eczematosa o con infección por hongos. Ulceras varicosas. Reaparición de várices en los pacientes tratados. La eliminación de las venas principales superficiales Ho previene la aparición de várices durante los embarazos posteriores. Sensibilidad a soluciones esclerosantes que puede causar muerte súbita (morruato de sodio). Complicaciones anestésicas y quirúrgicas. Problemas cosméticos: pigmentación de la piel. Induraciones perivenosas. Hemorragias petequiales, porque la presión venosa alta causa ruptura de los vasos pequeños. Pigmentación de la piel a causa de la hemosiderina de la sangre extravasada en ios tejidos.

\section{VIII.-Tratamiento}

A.-Tratamiento conservador-Las venas varicosas existentes previamente pueden ser agrabadas por el embarazo. Esos casos pueden aliviarse por el uso correcto de medias elásticas, las cuales son más útiles en la mujer embarazada que en otros pacientes, porque la duración de la obstrucción venosa es relativamente más corta (19).

Medias elásticas.-En casos moderados de várices, el uso de medias elásticas proporciona alivio por reducción de la presión venosa, haciendo que la sangre vaya hacia la circulación venosa profunda y ayudando a la circulación venosa hacia el corazón. El tratamiento de apoyo o soporte se obtiene con medias elásticas correctamente usadas. Una media elástica incorrectamente construída puede tener el mismo efecto indeseable de un vendaje incorrecto con variación en la presión a diferentes niveles. Inadecuada presión es, por consiguiente, inefectiva en la ayuda a las venas enfermas. Presión excesiva puede ser perjudicial, ya que disminuye ia circulación arterial y la venosa. Una combinación de las dos anteriores es más perjudicial, porque, un exceso de presión en un punto dado corta la circulación venosa y aumenta la dilatación 
venosa por debajo de la región comprimida (13). Medias elásticas impropiamente hechas o usadas pueden agravar la condición que tratan de corregir. En general, se puede decir que la presión debe aisminuir del tobillo hacia los muslos y que ciertas relaciones deben existir entre las presiones en los tobillos, pantorrillas y muslos con el objeto de proporcionar máxima efectividad y seguridad. El diámetro de la pierna cambia marcadamente del tobillo hacia el muslo, aumentando en la pantorrilla y disminuyendo nuevamente en la rodilla. Por consiguiente, la media elástica ideal debe construírse de acuerdo con presiones establecidas previamente (13). Bauer \& Black producen diversas medias elásticas ideales, en estilos por debajo de la rodilla o por encima de ésta, con pie cerrado o abierto, que son efectivas y bien construídas. Son hechas de nylon y no sabemos qué tan bien toleradas sean en cli_ mas calientes, en donde las medias de algodón son más confortables. Para comprar una media elástica correcta las medidas deben ser tomadas por el vendedor, en el muslo, la rodilla, la pierna y el tobillo. La longitud de la pierna también se debe tomar. Por referencia a unos patrones, con esas medidas, la media elástica adecuada para cada caso es obtenida. Instrucciones escritas son dadas a cada paciente con cada par de medias, tocantes al método propio de aplicación, lavado y otros cuidados. Es muy importante usar una media elástica correctamente.

Vendajes elásticos.-La aplicación correcta de un vendaje elástico es importante, comenzando por el pie hacia arriba, teriendo cuidado de que las vueltas sean mantenidas y de que presión igual pero moderada sea hecha en todo el miembro. La presión de la vuelta de encima no debe ser mayor que la de la vuelta inferior, porque en ese caso las venas por debajo de la vuelta apretada del vendaje necesariamente se dilatan. No es necesario usar el vendaje durante la noche, cuando el paciente está acostado, pero debe ser puesto antes de lavantarse de la cama, en la mañana.

B. Tratamiento radidal.-El tratamiento radical para las venas varicosas permanece en controversia. Inyecciones esclerosantes y la operación de arrancamiento tienen algunas indicaciones.

a) Inyecciones esclerosantes.-Muchas técnicas de inyecciones esclerosantes para la vena safena han sido presentadas desde la invención de la jeringa hipodérmica en 1853. El objeto es obstruir permanentemente las venas tratadas, por medio de trombosis ocasionada por flebitis química. Si bien hay obstrucción inmediata, 
dentro de un mes y más de cinco años la recanalización se efectúa y las várices vuelven a hacerse presentes, estando entonces las válvulas permanentemente dañadas por adhesión o por absorción, y la vena se ha hecho definitivamente incompetente. Inyecciones esclerosantes deben ser usadas solamente para várices muy pequeñas; pero, como se dijo previamente, no son permanenteinente satisfactorias para grandes várices. La dosis promedio es de 2 a 4 c. c. de una solución al $5 \%$ de morruato de sodio o de una al 3,5\% de ricinoleato de sodio en pacientes sensitivos al morruato. La sensibilidad al morruato de sodio puede ocasionar muerte súbita. Es por consiguiente prudente que el paciente sea chequeado con una dosis de 0.5 a 1 . c. c. intravenoso, 12 a 24 hoIas antes de iniciar el tratamiento. El tetradecil sulfato de sodio en solución del 1 al 3\% parece ser una mejor solución esclerosante. Las inyecciones deben aplicarse diariamente. Si la reacción iocal es severa, 2 c. c. deben aplicarse dos veces al día. Con la apazición de una reacción sistémica inyecciones posteriores no deben aplicarse.

Para inyectar una vena pequeña sin previa ligadura, el paciente debe pararse sobre una mesa en la mejor posición para que el médico coja la vena. La inyección se hace generalmente con una jeringa de 2 a 5 c. c. y con una aguja número 25. Es importante que la aguja quede libre dentro de la luz de la vena, para aue los tejidos que la rodean no reciban la substancia irritante. Por consiguiente, debe hacerse aspiración con el émbolo de la jeringa y estar seguro de que la sangre fluya fácilmente, antes de inyectar la solución. Cualquier abultamiento local de la piel durante la inyección, indica que la solución se está aplicando en los tejidos y que debe suspenderse inmediatamente y ponerse un vendaje apretado en el área afectada.

Generalmente el paciente se queja de dolor si la solución es aplicada en los tejidos. La inyección de 1 o 2 c. c. de solución salina fisiológica, puede diluir la solución esclerosante inyectada en ios tejidos, si se considera necesaria. También se puede infiltrar la región afectada con procaina en solución al 1 o $2 \%$. Después de ia inyección esclerosante, un apósito de gasa estéril es aplicado en el sitio de la inyección y debe mantenerse presión moderada con un vendaje elástico por dos o tres horas (4). Después, una media elástica puede estar mejor indicada, sin el apósito de gasa que contraindicaría el uso de ésta.

El valor de la ligadura de la vena safena-Inyecciones esclerosantes sin ligadura de la vena, en donde la circulación de retor- 
no esté presente, están definitivamente contraindicadas. Ligadura alta de la vena safena, solamente o con inyección retrógrada al tiempo de la ligadura, es un procedimiento inadecuado (6), (8). Más de $50 \%$ de los pacientes tratados con ligadura alta de la vena safena, con o sin múltiples interrupciones distales, asociadas con inyecciones esclerosantes, presentaron reincidencia dentro de ocho años. La tendencia a presentar venas varicosas es gradualmente progresiva.

b) Tratamiento radical quirúrgico.-Condiciones para flebectomía en el embarazo:

1. El embarazo por sí solo no es una contraindicación para la extirpación radical de venas varicosas (11), (16).

2. Várices existentes antes del embarazo (7).

3. Demostración de incompetencia del sistema de la vena safena y de competencia de la circulación venosa profunda, por una - más de las pruebas o tests aceptados (7).

4. Solicitud del paciente para estética y alivio (7). Pacientes con várices marcadas de los miembros inferiores y con várices de la vulva deben recibir alivio por medio de la cirugía (16). Con frecuencia, si el paciente insiste en algo definitivo o si los síntomas son severos debe practicarse arrancamiento de las várices. En tales casos, la rama superficial de la vena pudenda externa debe extirparse tan lejos como sea posible, dentro del labio, para eliminar las várices vulvares. Esto debe también hacerse en cada mujer durante la edad adulta, cuando tenga historia previa de várices vulvares (10).

5. Presencia de una o más manifestaciones subjetivas (7).

6. Prevención de trombosis y de embolía pulmonar. Las venas varicosas son uno de los más importantes factores predisponentes de la enfermedad trombo-embólica (11). De 52 pacientes con várices tratadas conservadoramente, 21 presentaron posteriormente tromboflebitis y 2 presentaron embolía pulmonar no fatal. De los 14 pacientes tratados quirúrgicamente, solamente uno presentó trombo-flebitis y ninguno presentó embolía pulmonar (8).

7. La flebectomía está especialmente indicada en esos pacientes con complicaciones de dermatitis por éstasis venosa, ulceraciones, edema, induración celular y trombosis venosa superficial aguda y en esas pacientes con historia de problemas durante un embarazo anterior con hemorragias por várices vulvares o con trombosis venosa del puerperio. 
8. La operación de arrancamiento, a causa de la reincidencia de las várices durante los embarazos posteriores, está solamente indicada en mujeres que proyectan no tener más hijos (16).

\section{IX.-Técnica de la operación de arrancamiento (stripping) (10)} ácido pirogálico, fórmula:

Previamente a la operación, las várices deben identificarse por marcas en la piel: a) con una solución débil de nitrato de plata al $15 \%(9)$; b) con lápiz indeleble; c) con solución indeleble de Solución de ácido pirogálico . . . . . . . . . 5 gramos

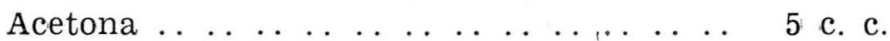

Solución de cloruro férrico N. F. . . . . . . 40 c. c.

Alcohol etílico c. s. p. .. . . ... . . . . . . 100 c. c.

Guardarla en frasco negro. No exponerla excessivamente al aire. No usarla después de 10 días; d) o por marcas o rasguños en ia piel con una aguja hipodérmica, bajo condiciones de asepsia. Este método es relativamente indoloro y bien tolerado. La pierna debe afeitarse, cepillarse con agua y jabón y pintarse con una so'ución antiséptica.

Se puede usar anestesia general, raquídea, raquídea fraccionada o caudal. La anestesia espinal o raquídea es preferida por su inocuidad para el feto (16). Para acortar el tiempo operatorio y reducir el período de anestesia, dos equipos de cirujanos trabajando simultáneamente son deseables, si las várices son bilaterales (16). El promedio de duración de la operación es de dos horas (16).

El segundo trimestre del embarazo es el mejor para la cirugía electiva, porque $86 \%$ de los abortos se efectúan antes de la doceava semana del embarazo, y por la posibilidad de iniciar el parto en el embarazo avanzado, y porque en el tercer trimestre la hemorragia es mayor a causa de la mayor dilatación venosa. Está establecido que un $77 \%$ de las várices se desarrollan en los dos primeros trimestres y se ha encontrado que la cirugía es raramenté necesaria después del séptimo mes (16).

Una incisión vertical de 5 a 7 centimetros de larga, dependiendo del espesor de la capa de grasa, justamente sobre la arteria femoral, la cual se identifica por palpación, comenzando en el pliegue inguinal (Ligamento de Poupart), permite un campo mejor que una incisión paralela a él. La vena safena larga se identifica en la fosa oval. Esta vena y todas sus tributarias en esta área se disecan: pudenda externa superficial, fémoro-cutánea la 
teral, epigástrica superficial, circunfleja ilíaca superficial. Las tributarias se seccionan individualmente entre pinzas y se ligan con catgut crómico 0 o con seda, cerca de la femoral común, para evitar formación de trombos si se deja un muñón largo (7), (16), (18).

Un extirpador o arrancador flexible es introducido dentro de la vena hasta donde sea posible hacia abajo. Numerosos extirpadores se pueden obtener: Myrs intraluminal, Babcock intraluminal, Mayo extraluminal, Williams-Linton, etc. La vena se liga sobre el extirpador. El extirpador es halado hacia abajo a través de una obertura hecha en el extremo terminal de la vena, mientras con la otra mano se va aplicando presión sobre la piel del muslo. Si la luz de la vena no permite el paso del extirpador intraluminal, in extirpador extraluminal se debe emplear (14). La vena puede arrancarse completamente hacia abajo hasta el dorso del pie, en dos o tres incisiones. La sacada del alambre extirpador invierte la vena, la cual es extraída a través de su luz. Ocasionalmente, cuando la vena es extremadamente larga y tortuosa, excisión de varios segmentos debe combinarse con arrancamiento. La fascia superficial se sutura con catgut crómico 00, el tejido subcutáneo con catgut 00 y la piel con puntos interrumpidos de seda.

Una forma retrógrada parecé más fácil: el tronco terminal de la vena safena larga se secciona a nivel de la parte media del maléolo interno y se arranca del tobillo hacia la ingle. Haciendo la ¿ncisión original a nivel del tobillo, y pasando el extirpador intraluminal hacia arriba, es una ayuda para localizar fácilmente el tronco de la vena safena, palpando la punta, en forma de oliva, del extirpador. Arrancamiento de la vena del tobillo hacia ia ingle', con ligadura de las venas comunicantes por múltiples incisiones, realzadas por el procedimiento de los dos equipos de cirujanos, parece ser el procedimiento quirúrgico más satisfactorio (18).

La vena safena corta es arrancada como en el procedimiento para la vena safena larga. La incisión original se hace equidistante entre el maléolo externo y el tendón de Aquiles. El extirpador es pasado hacia arriba hasta cerca de la unión safeno-poplítea, en la fosa o espacio polplíteo inferior, entre las cabezas de los dos músculos gastrocnemios, y se arranca hacia arriba. En la ligadura de la vena safena corta cerca del tobillo, se debe tener cuidado de no herir el nervio sural que pasa paralelo y junto a la vena safena corta en este sitio. 


\section{X.-Tratamiento post-operatorio}

Inmediatamente después de la operación de arrancamiento, presión con compresas gruesas es aplicada por 24 horas, sobre la parte media de la pierna, desde el tobillo hasta la ingle, usando dos compresas estériles dobladas y mantenidas por un vendaje elástico de 4 pulgadas, aplicado de el pie hacia arriba. Para prevenir ia trombosis venosa, el paciente se hace ambular en la tarde del día de la operación o el día siguiente a la operación y permanece hospitalizado solamente dos o tres días. El vendaje elástico se deie usar hasta que los puntos sean removidos en el séptimo día después de la operación. Entonces, subsecuentes inyecciones es:lerosantes (tetradecil sulfato de sodio en solución al 1 al 3\%) on iniciadas para las pequeñas ramas no eliminadas al tiempo de la cirugía, porque quirúrgicamente no es fácil seguir cada ra-ma hasta su terminación. La eficacia de cualquier tratamiento de las venas varicosas está en proporciónl directa con la habilidad cel cirujano para eliminar las venas perforantes incompetentes en cualquier parte que estén localizadas. Cada operación es un problema anatómico individual que debe ser resuelto por el cirujaso (14). Varios artículos han aparecido recientemente presentando series de pacientes varicosas tratadas quirúrgicamente durante el embarazo con excelentes resultados finales (11). La mejor medida en contra de las posibles recurrencias futuras es la cuidadosa erradicación quirúrgica de todas las venas incompetentes, en el muslo, la pierna y el pie, por arrancamiento combinado con disección y ligaduras (14).

\section{XI.-Angiectids}

Angiectid es el nombre dado a los vasos intradérmicos dilatados en los miembros inferiores durante el embarazo. Se deben diferenciar de las várices de la safena, que son dilataciones venosas subcutáneas. Las angiectids aparecen inicialmente durante el primer trimestre del embarazo, con más frecuencia en mujeres jóvenes y durante los primeros tres embarazos. Aproximadamente en la mitad de las pacientes, las angiectids desaparecen completamente antes de la sexta semana del post-parto. En la otra mitad los remanentes vestigios intradérmicos están en algunos casos asociados con cambios premenstruales y algo de dolor. Las várices de la vena safena están presentes antes del embarazo, crecen durante el embarazo y retornan al estado pregrávido después 
del parto en la mayoría de los casos. La angiectid es pequeña, superficial, intradérmica, saliente; es una agrupación de vasos azulados bien circunscritos que tienen predilección por ciertos sitios específicos, generalmente en la cara interna de la pantorrilla. La angiectid es generalmente caliente y delicada. El dolor causado por la angiectid está en contraste con la pesantez y fatiga causada por las várices en la pierna. El dolor está presente en la mayoría de los casos y ocasiona siempre algo de incapacidad. Generalmente es lo suficientemente severo para impedir que el paciente esté de pies por más de cinco minutos. Cuando el dolor está presente, la angiectid es caliente, sensible y tensa. Estas características están limitadas a la angiectid y no comprenden los tejidos que la rodean. Con excepción de las angiectids, el sistema venoso de la paciente es generalmente normal. Las pacientes con angiectids tienen títulos bajos o subnormales de estrógenos en el suero y del pregnanediol urinario. El paciente con várices de la vena safena las cantidades de estrógeno y de pregnandiol son normales. Los 17 ketosteroides urinarios y la gonadotropina coriónica sérica en las pacientes con várices de la vena safena o con angiectids, son normales. Las funciones hepática y renal, los niveles de calcio en la sangre y los tiempos de sangría, de coagulación y la protrombina, están dentro de los límites normales en pacientes con angiectids. Desde que la mayoría de las pacientes con angiectids tienen una deficiencia en estrógenos, el tratamiento con estrógenos se ha encontrado efectivo para aliviar el dolor, la incapacidad, la sensibilidad y la tensión de las angiectids. Dietilstil-bestrol en dosis diarias variables entre 50 y 150 miligramos han probado ser efectivas (15). Los estrógenos tienen una acción vasodilatadora solamente sobre los capilares y las vénulas (15).

\section{XII.-Conclusiones}

Las venas varicosas y las angiectids son agravadas por embarazos frecuentes. El tratamiento de las várices en las mujeres embarazadas es un problema no solamente desde el punto de vista del alivio de la paciente y de los problemas cosméticos, sino tamoién en consideración a la profilaxis de la complicación tromboembólica. El tratamiento quirúrgico permanece en controversia (4), (8). El tratamiento de las venas varicosas durante el embarazo es más difícil y requiere más tiempo que en las no embarazadas. Los resultados satisfactorios en la cirugía de las várices en cualquier situación dependen principalmente de la cantidad de renas extirpadas. 
El estado de embarazo de la paciente le permite al cirujano alguna ventaja desde el siguiente punto de vista: con la dilatación del tronco principal y de sus ramas y particularmente con ia dilatación de las venas comunicantes que se han hecho incompetentes, más fácil eliminar todas las venas que están causando el problema, o que pueden permanecer dilatadas después que el embarazo termina. El procedimiento de arrancamiento es el más satisfactorio en embarazadas y en no embarazadas con várices sintomáticas y puede practicarse con seguridad y con resultados tan permanentes como en las no embarazadas. Recurrencias envolviendo nuevos paquetes de venas son comunes después de todos los procedimientos, por lo tanto, es conveniente no ofrecerle - la paciente una "garantía de por vida" de que no tendrá más várices.

Preguntas que los médicos deben contestar en relación con medias y vendajes elásticos fueron expuestas en este trabajo. Descanso en la cama y elevación del miembro o miembros inferiores alivia los síntomas. Actualmente, como en el pasado, el tratamiento de las várices ha sido dirigido más hacia los efectos mecánicos de las várices que hacia las causas básicas que ocasionan la dilatación de las venas y su irregularidad. Es solamente en la actualidad que se están investigando factores en relación con la etiología de las várices, y es de esperarse que algún día la cuchilla de los cirujanos ya no sea necesaria.

Nosotros no podemos ofrecerle al paciente varicoso completo y satisfactorio tratamiento, pero podemos decir que millones de pacientes pueden ser aliviados, que la mayoría de las complicaciones pueden ser prevenidas, y que un gran porcentaje de los que nos consultan pueden ser curados. Facilidades para servicios especializados en várices faltan aún en muchos hospitales. Hasta que las várices logren la misma importancia que a modo de comparación tienen las fracturas, muy poco progreso se habrá logrado (Foote) (5). 


\section{XIII.-Referenciูas}

1. Kampmeier, O. F., and Birch, C. L. F., The origin and development of the venous valves; with particular reference to the saphenous district. Am. J. of Anat., 38: 451-499. 1927 (University of Illinois, Chicago).

2. Adams, J. C., Etiological factors in varicose veins of lcwer extremities. Surg. Gyn. Obst., 69: 717-725. 1939 (Univ. of Oregcn Medical School, Portland, Oregon).

3. Vea!, J. R., and Hussey, H. H.: The venous circulation in the lower extremities during pregnancy. Surg. Gyn. Obst., 72: 841-847. 1941 (Gallinger Minicipal Hospital, and Georgetown University Schcol of Medicine, Washington, D. C.).

4. Sherman, R. S., Varicose veins; anatomic findings and a operative procedure based upon then. Ann, of Surg., 120: 77.2-784, 1944 (University of California Medical Sshool. San Francisco, Calif.).

5. Lyall, D. The treatment of varicose veins. Surg. Gyn. Obst., 82: 332-341. 1946 (New York, N. Y.).

6. Wright, I. S., Vascular Diseases in Clinical Practice. The Year Bock Publishers Inc. 304 South Dearbon Street, Chicago, 1948, p. p. 20-91 and 427-441 (Cornell University Medical College \& New York Hospital).

7. Foote, R. R., Varicose veins. Harrow Hospital, London. The C. V. Mosis Company, Sî. Louis, M॰., 1949 (1954).

8. Peyton, F. W., and Loop, F. A., Saphenous phlebectomy for varicose veins during pregnancy. Am. J. Obst. Gyn., 58: 318-325, 1949 (St. Elizabet Hospital, Lafayctte, Ind.).

9. Sherman, R. S., Varicose veins; furter findings based on anatomical and surgical dissections. Ann. of Surg., 130: 218-232, 1949 (University of Califcrnia Medical School, San Francisco, Calif.).

10. Quattlebaun, F. W., and Hodgson, J. E., Surgical treatment of varicose veins in pregnancy. Surg. Gyn. Obst., 95: 336-340, 1952 (St. Paul, Minnesota).

11. Basmajian, J. V., The distribution of valves in the femoral, external iliac, and common iliac veins and their relationship to varicose veins. Surg. Gyn. Obst., 95: 537-542, 1952 (University of Toronto, Canadá).

12. Venous Disorders of the Lower Extremity. Ciba Clinical Symposia. †ol. 5. No 4, July, August, 1953 (Summit. New Jersey).

13. Cavanach, M. J., and Weinberg, P. C.: The treatment of varicose veins auring pregnancy. U. S. Armed Forces M. J., 5: 1619-1622, 1954 (U. S. Air Force Hospital, Sheppard Air Force Base, Tex.).

14. Williams, A. F.: A comparative study of the venous valves in the limbs. Surg. Gyn Obst., 99: 676-678, 1ऽ54 (Manchester, England).

15. Elastic stccking compression in the therapy of varicose veins. Bauer \& Black, The Kendall Company 309 West Jackson Boulevard, Chicago 6, Ill. 1956. 
16. Fried, P. H., Perilstein, P. K., and Wagner, F. B., Jr.: The hormonal cause of the so-called varicose veins of pregnancy. A. M. A. Archives of surgery, 73: 253-261, 1956 (Jefferson Medical College and Hospital, Philadelphia).

17. Lofgren, K. A., Myers, T. T., and Webb, W. D., Jr.: Recurrent varicose veins. Surg. Gyn. Obst., 102: 729-735, 1956 (Mayo Clinic and Mayo Foundaion, Rochester, Min.).

18. Weisman, R. E., and Hanover, N. H.: Saphenous vein stripping for varicose veins during pregnancy, J. A. M. A., 161: 1459-1462. 1956 (Dartmount Medical School and Medical Center).

19. Operations for varicose veins: British Medical Journal. London. № 5020 , p. p. $692-693,1957$.

20. Evoy, M. H.: Varicose veins and pregnancy. Northwest Medicine (Seattle), 56: 318-319, 1957 (uncensulted).

21. Vaughn, A. M., Annan. C. M., and Caserta, J. A.: A combined twotean technic in the surgical treatment of varicose veins. Am J. of Surgery. 94: 97-101, 1957 (Stritch School of Medicine of Loyola Univ., Mercy and County Hospital, and the Vaughn Medical Group, Chicago, I11.).

22. Fccte, R. R.: Varicose vein problems in general practice. Practitioner, Iondon, 179: 59-66, 1957 (unconsulted). 